\title{
DOR MIOFASCIAL E FIBROMIALGIA: DE MECANISMOS ETIOLÓGICOS A MODALIDADES TERAPÊUTICAS
}

\section{MYOFASCIAL PAIN AND FIBROMYALGIA: FROM ETIOLOGIC MECHANISMS TO CLINICAL MANAGEMENT}

\author{
Rosangela Seiko Seó ${ }^{1}$, Nara Hellen Campanha ${ }^{2 *}$, \\ Francisco Guedes Pereira Alencar Júnior ${ }^{3}$, Karin Hermana Neppelenbroek ${ }^{2}$, \\ Hércules Jorge Almilhatti ${ }^{4}$ \\ ${ }^{1}$ Centro Universitário de Várzea Grande - UNIVAG, Curso de Odontologia, Várzea Grande, MT \\ $2^{*}$ Autor para contato: Universidade Estadual de Ponta Grossa - UEPG, Departamento de \\ Odontologia, Campus em Uvaranas, Ponta Grossa, PR; (42) 3220-3106; \\ e-mail: narahell@uepg.br \\ 3 Universidade Estadual Paulista "Júlio de Mesquita Filho" - UNESP, Faculdade de \\ Odontologia, Departamento de Materiais Odontológicos e Prótese, Araraquara, SP \\ ${ }^{4}$ Universidade Federal do Paraná - UFPR, Departamento de Odontologia, Curitiba, PR
}

Recebido para publicação em 23/11/2006

Aceito para publicação em 23/04/2007

\section{RESUMO}

Fibromialgia é uma doença crônica, complexa, caracterizada pelo desconforto músculo-esqueletal generalizado, alterações no sistema nervoso central, endócrino e alterações psicológicas dos pacientes. Dor miofascial é uma desordem muscular envolvendo um ou mais músculos que apresentam trigger points (pontos de gatilho) miofasciais, acompanhada por dor local e referida, diminuição da amplitude de movimento, fraqueza e, freqüentemente, fenômenos autonômicos. Neste trabalho foram revisados os possíveis fatores etiológicos da fibromialgia e dor miofascial, diagnóstico diferencial e tratamentos recomendados, e ainda discutiu-se a participação dos profissionais da área de saúde no plano de tratamento. Esses profissionais exercem uma importante função no diagnóstico e tratamento dessas enfermidades, visto que ambas podem coexistir e confundir-se com outras desordens que acometem a região de cabeça e pescoço, evitando, assim, intervenções médicas ou odontológicas desnecessárias. Além disso, devido ao acometimento do sistema nervoso central, a fibromialgia pode perpetuar a dor miofascial bem como outras desordens temporomandibulares coexistentes. Devido à sua complexidade e multifatoriedade, o sucesso no tratamento da fibromialgia e da dor miofascial requer a instituição de diversas modalidades terapêuticas, associadas à participação de uma equipe profissional multidisciplinar.

Palavras-chave: fibromialgia, dor miofascial, doenças musculares, dor crônica, pontos dolorosos, pontos de gatilho miofasciais 


\begin{abstract}
Fibromyalgia is a complex chronic disease which is often associated with widespread musculoskeletal pain and altered function of the central nervous system, neuroendocrine disorders, and psychological distress. Myofascial pain is a pain disorder involving at least one muscle which presents myofascial trigger points, local and referred pain, diminished range of motion, stiffness and autonomic phenomena. The aim of this study was to review the etiological factors of fibromyalgia and myofascial pain, exclusion diagnosis, suitable treatments and, additionally, the role of health professionals in the treatment plan. These professionals play an important role in the diagnosis and treatment of fibromyalgia and myofascial pain, as these diseases often coexist and/or combine with other head and neck diseases. Thus, they would avoid unnecessary interventions. Moreover, the altered function of the central nervous system may contribute to or perpetuate the myofascial pain and/or other temporomandibular disorders. Because of their complex and multifactorial features, a multidisciplinary therapeutic approach with a team of health professionals and different treatment modalities should be applied in order to assure a more effective management of fibromyalgia and myofascial pain and associated disorders.
\end{abstract}

Key words: fibromyalgia, myofascial pain syndromes, muscular diseases, painful points, trigger points, chronic pain

\section{Introdução}

Durante muitos anos, a fibromialgia (FM) foi considerada como uma desordem músculo-esqueletal. Simons (1986) sugeriu que FM e dor miofascial (DMF) poderiam ser diferentes interpretações de uma mesma condição. Além disso, foi encontrado um alto índice de coexistência dessas duas enfermidades em um grande número de pacientes (Wolfe et al., 1990).

A FM e a DMF apresentam muitas características em comum, tais como a presença de dor, desconforto músculo-esqueletal, alta prevalência em mulheres e influência do SNC. Os pacientes acometidos por ambas as enfermidades também podem apresentar distúrbios do sono, depressão, ansiedade, estresse e baixo condicionamento físico. Esses fatos, associados à ausência de testes laboratoriais específicos para uma e outra doença, contribuem para que, clinicamente, exista uma dificuldade considerável em se diagnosticar ambas as condições (Yunus et al., 1988). No presente trabalho, os possíveis fatores etiológicos da FM e DMF, diagnóstico diferencial e tratamentos recomen- dados para essas enfermidades foram revisados.

\section{Revisão da literatura}

\section{Fibromialgia}

\section{Definição e características clínicas}

A Fibromialgia (FM) é uma doença crônica, complexa, caracterizada pelo desconforto músculoesqueletal generalizado e alterações nos sistemas nervoso central, endócrino e alterações psicológicas do paciente (Neek et al., 2002). Contudo, ela não deve ser considerada uma desordem músculo-esqueletal visto que não há uma disfunção localizada, embora existam alterações celulares nas fibras musculares causadas pelas alterações bioquímicas relacionadas à doença (Yunus et al., 1988). Essa entidade patológica é definida como síndrome por apresentar um conjunto de sinais e sintomas que caracterizam a doença. O sintoma ca- 
racterístico da FM é a dor generalizada localizada simetricamente em ambos os lados do corpo, freqüentemente acompanhada de fadiga, distúrbios do sono e presença de pontos dolorosos. Outros sinais e sintomas incluem depressão e ansiedade; parestesias; dores de cabeça (cefaléia do tipo tensional e enxaqueca); dismenorréia; síndrome do intestino irritado; síndrome da bexiga irritada; dormência e formigamento recorrente envolvendo extremidades superiores e inferiores; secura nos olhos e boca; hiperalgesia e alodínia; tonturas e disfunção cognitiva (dificuldades de recordar eventos, processar informações e realizar tarefas) (Yunus et al., 1988; Staud e Domingo, 2001; Alencar Jr, 2002; Mehendale e Goldman, 2002)

Estima-se que a FM afeta de 2 a $7 \%$ da população mundial, sendo mais prevalente em mulheres do que em homens em uma proporção de 9:1 (Staud e Domingo, 2001). Essa desordem atinge mais frequentemente pessoas entre 45 a 60 anos, porém sua prevalência aumenta com a idade (Sprott, 2003). Embora incomum, essa síndrome pode acometer crianças e adolescentes (Buskila, 2003).

Considerando que a FM pode ser confundida com outras doenças, uma vez que muitos sintomas podem ser encontrados em outras patologias, em 1990, aAmerican College of Rheumatology adotou como critério de classificação (Wolfe et al., 1990) a presença de dor crônica generalizada (em ambos os lados do corpo, acima e abaixo da cintura) por pelo menos 3 meses e o relato de dor, em pelo menos 11 de 18 pontos dolorosos previamente descritos, durante a palpação digital, aplicando-se uma força de $4 \mathrm{~kg} / \mathrm{cm}^{2}$. O diagnóstico diferencial da FM inclui polimialgia reumática, hipotireoidismo e artrite reumática (Yunus et al., 1988; Ferucci e Collier, 2002). Outras doenças menos comuns podem ser incluídas, tais como: lupus eritematoso sistêmico, miopatias inflamatórias, hipertireoidismo, infecções (HIV, EBV, Doença de Lyme) e osteomalacia (Ferucci e Collier, 2002). Visto que há outras condições clínicas que podem ser confundidas com a FM, alguns testes laboratoriais devem ser realizados (contagem sanguínea; urianálise; creatina quinase; fator reumatóide; anticorpo antinuclear; testes de função da tireóide e velocidade de eritrosedimentação) para a exclusão de tais condições (Ferucci e Collier, 2002). No entanto, é importante ressaltar que a FM não apresenta evidência laboratorial.

\section{Fisiopatologia}

A fisiopatologia dessa doença é bastante complexa, sendo evidenciadas várias alterações fisiológicas, que geram muitas teorias a respeito de sua patogênese. Atualmente há uma tendência em considerá-la multifatorial, com fatores iniciantes e resultantes. Todos esses fatores podem também ser perpetuantes levando à cronicidade da doença (Alencar Jr, 2002; Russel, 1999).

Tem sido relatado que alguns pacientes possuem uma predisposição genética para o desenvolvimento da FM (Mehendale e Goldman, 2002; Jones et al., 2002; Buskila, 2003). Offenbaecher et al. (1999) avaliaram os genótipos da região promotora do gene transportador de serotonina (5-HTT) em pacientes com FM. Foi observada uma maior freqüência de genótipo S/S (5-HTT) em pacientes com FM quando comparados aos pacientes controles saudáveis. Os pacientes que apresentaram o subgrupo $\mathrm{S} / \mathrm{S}$ exibiram maiores níveis de depressão e estresse psicológico (Buskila, 2003). Embora controverso, alguns autores citam os traumas (físico e emocional) e algumas infecções como fatores predisponentes da FM (Alencar Jr, 2002; Mehendale e Goldman, 2002). Doenças infecciosas como o vírus Ebstein-Barr, HIV, hepatite viral e doença de Lyme são associadas ao desenvolvimento da FM (Staud e Domingo, 2001). Adicionalmente, traumas, como o abuso sexual, também foram relacionados à FM, embora sejam necessários estudos adicionais para confirmar essa hipótese (Staud e Domingo, 2001; Ferucci e Collier, 2002; Buskila, 2003). Atualmente a teoria mais aceita é a de que sua etiologia estaria relacionada com alterações no sistema neuroendócrinoimune-hormonal, distúrbios do sono e anormalidades do metabolismo muscular (McCarberg et al., 2003).

Adicionalmente, Neeck (2002) associou a sintomatologia da FM aos distúrbios psicológicos apresentados por esses pacientes. Nessa teoria, a regulação endócrina apresenta-se consideravelmente prejudicada, o sistema de feedback hormonal está alterado e ansiedade e depressão são os sintomas psicológicos mais proeminentes. Ademais, a dor crônica músculo-esqueletal deve ser mediada por um distúrbio no mecanismo responsável pelo processamento do estímulo da dor, reforçando ainda mais a hipótese de acometimento do SNC (Russel, 1999; Staud e Domingo, 2001). A con- 
seqüência clínica da sensitização central é reproduzida clinicamente pela hiperalgesia e alodínia, evidenciando um aumento na percepção da dor (McCarberg et al., 2003). A hiperalgesia é definida como uma resposta exagerada de dor aos estímulos nociceptivos e a alodínia é descrita como dor em resposta ao estímulo não nociceptivo, tal como um suave toque na pele. Ambas são freqüentemente observadas em pacientes com $\mathrm{FM}$. Concomitantemente, o fenômeno da neuroplasticidade deve perpetuar a alteração no funcionamento do mecanismo central ocasionando um ciclo vicioso e resultando na cronicidade da doença.

Além disso, as alterações hormonais e dos neuromediadores devem apresentar uma importante função na patogênese da FM (Neeck, 2002). Estudos têm demonstrado um nível elevado de substância P no fluído cerebroespinal e uma diminuição do nível sanguíneo de serotonina e triptofano em pacientes com FM. Especificamente, a serotonina estaria envolvida na estimulação na secreção do hormônio adrenocorticotrópico (ACTH) durante o estresse. A diminuição dos níveis de serotonina também está relacionada à redução do sono não-REM e ao aumento dos sintomas somáticos e da percepção de dor (Leventhal, 1999). Segundo Moldofsky (2002), os pacientes com FM apresentam alteração no sono não-REM. Assim, a interrupção do estágio 4 do sono não-REM é responsável pela pobre qualidade do sono (sono não reparador), rigidez matinal, fadiga e dor (Staud e Domingo, 2001). De acordo com Staud e Domingo (2001) e Neeck (2002), a secreção do hormônio do crescimento normalmente ocorre durante os estágios 3 e 4 do sono profundo (não-REM). Uma vez que esses estágios encontramse alterados, uma diminuição dos níveis de hormônio do crescimento tem sido observada. Essa diminuição da secreção do hormônio do crescimento estaria diretamente relacionada com a dor e fadiga muscular, visto que esse hormônio exerce importante função na manutenção e reparo muscular (Staud e Domingo, 2001).

Aproximadamente $30 \%$ dos pacientes com FM possuem problemas psicológicos, principalmente depressão (Tuzun et al., 2004) e muitos pesquisadores acreditam que essa não é a causa primária da doença (Alencar Jr, 2002; Ferucci e Collier, 2002). Segundo Yunus (1988) e Staud e Domingo (2001), o estresse poderia teoricamente induzir a ativação de vários neu- rônios hipotalâmicos, promovendo aumento dos níveis de ACTH e de cortisol, que estão associados com a depressão.

Alterações musculares, tais como hipoxia local, diminuição dos níveis de fosfato e bandas rígidas são observadas em pacientes com FM (Leventhal, 1999). Os músculos nas áreas dos pontos dolorosos apresentam-se histologicamente normais, porém com uma atrofia das fibras do tipo C. De acordo com Ferucci e Collier (2002), os pacientes com FM apresentam um maior gasto de adenosina trifosfato (ATP) durante a realização dos movimentos musculares normais quando comparados aos pacientes saudáveis. Foi relatada ainda, uma diminuição nos níveis de ATP, adenosina difosfato e fosforil creatina e um aumento nos níveis de adenosina monofosfato (AMP) e creatina, evidenciando a falta de condicionamento muscular desses pacientes. Segundo Buskila (2003), o edema da pele observado sobre as áreas dos pontos dolorosos deve estar associado a vasoconstrição local, reforçando a hipótese de hipoxia local na região de pontos dolorosos. Como a circulação arterial muscular local está reduzida, é provável que os músculos acometidos não recebam oxigênio suficiente para o metabolismo aeróbio. Assim, os metabólitos residuais não são removidos eficientemente, portanto, o reparo muscular deve ser afetado.

O prognóstico da FM é bastante variável e depende do grau de severidade da doença quando diagnosticada. No entanto, a completa remissão da doença é bastante rara (Forseth e Gran, 2002).

\section{Tratamento}

O tratamento da FM consiste na melhora das atividades funcionais, no controle da dor, da fadiga, das alterações de humor, melhora na qualidade do sono e reintegração psicosocial associados à doença, por meio das intervenções psicológicas (terapia comportamental-cognitiva), terapia física, terapia farmacológica e outras terapias alternativas (acupuntura, hipnoterapia, laser terapia, bio-feedback, etc) (Sprott, 2003).

O tratamento de escolha tem sido principalmente de natureza não farmacológica, pois somente poucas drogas têm demonstrado efetividade (Forseth e Gran, 
2002). Dentre os tratamentos não farmacológicos, a terapia psicológica tem demonstrado um papel importante no sucesso do tratamento da FM. O objetivo da terapia comportamental-cognitiva é ensinar ao indivíduo as práticas necessárias para o controle da dor por meio do aconselhamento, relaxamento e motivação. Além disso, a educação do paciente é importante para que aprenda a conviver com suas limitações. Nesse tipo de terapia, a natureza da síndrome deve ser explicada detalhadamente para o paciente. O paciente deve entender que a doença apesar de limitante não o impede de executar as funções diárias. Portanto, os pacientes devem ser encorajados a participar ativamente no tratamento de sua doença. Essas modificações no comportamento incluem orientações sobre a higiene do sono e o mecanismo da dor (Ferucci e Collier, 2002). Segundo Schaefer (2003), eventos estressantes, medicamentos, atividades fisicas, flutuações hormonais e ingestão de álcool e cafeína podem interromper o padrão do sono. O ciclo circadiano controla todas as funções dos ritmos fisiológicos, neuroendócrino e psicológico. Portanto, a regularização do ciclo circadiano torna-se primordial no tratamento da FM.

A maioria das pessoas com FM é sedentária e apresenta falta de condicionamento físico, associada à baixa resistência muscular e flexibilidade limitada. Assim, os objetivos da implementação de exercícios no plano de tratamento da FM são: manutenção da função durante as atividades diárias, diminuição da dor, fadiga e melhora na qualidade do sono (Geenen e Jacobs, 2001; Jones et al., 2002). No entanto, a eficácia da terapia física dependerá de muitas variáveis, tais como: a idade do paciente, o nível de condicionamento físico do paciente, a intensidade do exercício, a freqüência e a presença de fatores negativos (obesidade, artrite e presença de doenças de origem muscular) (Jones et al., 2002).

Dessa forma, todos os pacientes com FM devem ser tratados individualmente, ou seja, é importante que o profissional saiba e respeite o nível de tolerância individual de cada paciente aos exercícios propostos, evitando, assim, os microtraumas induzidos pela implementação de exercícios inapropriados, que resultam em um aumento na dor. Para a correta prescrição de exercícios, o profissional deve seguir os seguintes fundamentos: tempo, intensidade, duração e o tipo de exer- cício. O tempo indica a freqüência em que deve ser realizado o exercício; a intensidade envolve o grau de dificuldade imposta pelo exercício; a duração é a extensão da sessão dos exercícios e o tipo de exercício representa a modalidade de tratamento físico (aeróbico, resistência e alongamento). As intervenções aeróbicas incluem, ciclismo, caminhadas, dança aeróbica e exercícios aquáticos. Quando a prescrição do exercício resultar em aumento da dor, a intensidade dos exercícios deve ser diminuída antes de alterar sua freqüência e duração. Qualquer exercício deve ser realizado em intensidade e duração apropriadas para possibilitar a adaptação. Em geral, costuma-se prescrever apenas exercícios que os pacientes são capazes de realizar, facilitando a motivação e o sucesso da terapia (Jones et al., 2002). Para diminuir o risco de injúria e dor, os músculos a serem alongados devem ser aquecidos com movimentos ativos ou passivamente por meio da utilização de uma bolsa de água morna. Após aquecimento, o alongamento pode ser realizado várias vezes ao dia. O paciente deve ser orientado a alongar o músculo até sentir uma suave resistência, mantendo-o alongado por alguns segundos. É importante que o alongamento seja realizado sem dor para evitar os microtraumas. Com essa finalidade, a prática de hidroginástica em piscina aquecida facilita a fase de aquecimento dos músculos.

Com relação à última modalidade terapêutica a ser discutida, os antidepressivos tricíclicos são os agentes de escolha no tratamento farmacológico da síndrome fibromiálgica (Sprott, 2003). O efeito benéfico induzido pela Amitriptilina tem sido bem documentado e deve estar relacionado com sua capacidade de inibir a recaptação da serotonina e da norepinefrina (Forseth e Gran, 2002; Leventhal, 2002). Inicialmente, os antidepressivos tricíclicos são receitados em pequenas doses $(10 \mathrm{mg})$, antes de dormir e, posteriormente, sua dosagem deve ser aumentada até a dosagem máxima de 50-100 mg antes de dormir (Ferucci e Collier, 2002). Os efeitos colaterais relacionados ao medicamento são: ganho de peso, constipação, hipotensão ortostática e agitação (DEF, 2001/02). De acordo com Leventhal (Leventhal, 1999) a utilização constante desse fármaco não é indicada devido ao risco de taquifilaxia. Isto normalmente ocorre após 2 ou 3 meses de uso contínuo dessa droga, porém, a suspensão do medicamento por 2 a 4 semanas é o suficiente para 
restaurar a eficácia da droga.

A ciclobenzaprina apresenta propriedades similares aos antidepressivos tricíclicos, portanto, a dosagem recomendada é a mesma. A medicação deve ser receitada em pequenas doses $(10 \mathrm{mg})$ antes de dormir e, em seguida, aumentada até no máximo $40 \mathrm{mg}$, de acordo com a necessidade (Ferucci e Collier, 2002). As reações adversas observadas com maior freqüência são: sonolência, secura na boca e vertigem. As reações relatadas em $1 \%$ a $3 \%$ dos pacientes foram fadiga, debilidade, astenia, náuseas, constipação, dispepsia, sabor desagradável, visão turva, cefaléia, nervosismo e confusão (DEF, 2001/02).

Outra droga utilizada no tratamento da FM é a Fluoxetina $20 \mathrm{mg} /$ dia, responsável pela melhora no distúrbio do sono e depressão (Leventhal, 1999). A medicação ajuda os pacientes com depressão pelo aumento na disponibilidade de serotonina no cérebro. Talvez por serem específicos na recaptação de serotonina são mais eficazes para o tratamento da depressão do que para a dor em si. Sendo assim, esse medicamento tem demonstrado maior efetividade quando associado ao antidepressivo tricíclico (Amtriptilina) (Forseth e Gran, 2002).

Dentre os analgésicos utilizados para o tratamento da FM, o Tramadol tem demonstrado ser a melhor alternativa quando comparado aos analgésicos opióides convencionais (Mehendale e Goldman, 2002). O Tramadol é um combinado análogo ao opióide que inibe a noreprinefrina e libera a serotonina (Forseth e Gran, 2002), promovendo o alivio da dor em pacientes com FM (Ferucci e Collier, 2002; Forseth e Gran, 2002). O Tramadol é comercializado em caixas contendo 10 cápsulas de $50 \mathrm{mg}$ (DEF, 2001/02), sendo receitada com uma dosagem que varia entre 50-400 $\mathrm{mg}$ /dia por 3 semanas (Leventhal, 1999; Forseth e Gran, 2002). Os efeitos colaterais relacionados a essa droga são: náuseas e tontura (DEF, 2001/02).

Atualmente, estão sendo testadas drogas alternativas tais como o Oxitriptofano, o hormônio do crescimento e a relaxina. O oxitriptofano é um metabólito intermediário do aminoácido essencial L-triptofano na biossíntese da serotonina, sendo facilmente absorvida por via oral (Forseth e Gran, 2002). A administração terapêutica do Oxitriptofano tem demonstrado eficácia quando administrado em doses de $300 \mathrm{mg}$ (via oral) por 90 dias ou $100 \mathrm{mg}$, três vezes ao dia, por 30 dias (Leventhal, 2002; Forseth e Gran, 2002).

O hormônio do crescimento, como comentado anteriormente, está intimamente relacionado com os estágios 3 e 4 do sono profundo (não-REM). No entanto, esse estágio apresenta-se freqüentemente alterado em pacientes com FM, gerando uma diminuição da secreção desse hormônio. Tendo em vista que esse hormônio é de fundamental importância na manutenção e reparo muscular, a reposição hormonal tem demonstrado benefícios aos pacientes com FM (Staud e Domingo, 2001; Neek, 2002).

A Relaxina é um hormônio produzido primariamente pelo corpo lúteo, placenta, útero e possivelmente no cérebro. Porém, sua presença é detectável (quantitativamente) apenas durante a gravidez. Foi sugerido que os pacientes com FM apresentam alterações nos hormônios sexuais (Neek, 2002). Segundo Alencar Jr. (2002), esse hormônio poderia promover um aumento na microcirculação no interior dos músculos, aumento na produção do hormônio do crescimento, melhora na qualidade do sono e remodelação muscular, dos ligamentos e tendões. Contudo, estudos adicionais são necessários para avaliar a eficácia de tal hormônio no tratamento da FM.

Além disso, a instituição de terapias alternativas como Bio-feedback; TENS; acupuntura; laser terapia; terapia a frio e calor e hipnoterapia tem demonstrado resultados positivos para alguns sintomas da fibromialgia, porém, devido à falta de estudos controlados, estudos adicionais são necessários para comprovação de tal eficácia (Forseth e Gran, 2002; Leventhal, 2002).

\section{Dor Miofascial}

\section{Definição, características clínicas e fisiopatologia}

A dor miofascial $($ mio $=$ músculo; fascial $=$ relativo a fáscia) é uma desordem dos músculos esqueléticos que apresenta elevada prevalência na população, uma vez que atinge quase todo indivíduo pelo menos uma vez durante a vida (Travell e Simons, 1999). Os músculos (tendões ou fáscias) afetados por essa desordem dolorosa contêm algumas fibras musculares apresentando bandas rígidas perceptíveis à palpação, as 
quais, por sua vez, contém no seu interior os trigger points (pontos de gatilho). Os trigger points são regiões nodulares de contração muscular localizada capazes de produzir dor local e referida (sentida à distância do local de origem da dor), limitações dos movimentos e/ou efeitos excitatórios centrais secundários autonômicos (Han e Harrison, 1997; Alvarez e Rockwell, 2002). Apesar de sua alta prevalência, principalmente em mulheres, o diagnóstico da dor miofascial (DMF) é muitas vezes negligenciado e, conseqüentemente, o clínico torna-se incapaz de instituir a correta terapia. Até os dias de hoje, o diagnóstico da DMF baseia-se tão somente no exame físico e na anamnese e não conta com exames laboratoriais ou radiográficos (Borg-Stein e Simons, 2002).

A DMF tem uma grande prevalência entre os indivíduos que se apresentam com queixa de dor regional. A prevalência varia de $21 \%$ dos pacientes encontrados em uma clínica de ortopedia geral a 30\% dos pacientes de uma clínica médica geral com dor regional e até prevalências altas como 85 e $93 \%$ dos pacientes que se apresentaram a centros especiais de tratamento de dor (Borg-Stein e Simons, 2002).

A DMF é uma desordem muscular local, e sob compressão digital, os trigger points podem reproduzir a queixa de dor ou outros sintomas relatados pelos pacientes (Han e Harrison, 1997; Alvarez e Rockwell, 2002).

Algumas vezes, os profissionais de saúde podem negligenciar a presença de trigger points e realizar o diagnóstico incorreto da causa de dor dos pacientes. De acordo com Travell e Simons (1999), alguns dos diagnósticos não raros em negligência à $\mathrm{DMF}$ são: angina pectoris atípica, apendicite, angina atípica, neuralgia facial atípica, enxaqueca atípica, dores nas costas, dor crônica na parede abdominal, dismenorréia, dor de ouvido, epicondilite, dor de cabeça occiptal, neuralgia pós-herpética, radiculopatia, síndrome escapulocostal, bursite, desordem temporomandibular articular, dor de cabeça tensional e síndrome de Tietze.

Hipoteticamente, a formação dos trigger points se dá por meio da fadiga de fibras musculares de músculos que foram muito exigidos, com conseqüente encurtamento e deficiência de suprimento de oxigênio e nutrientes, aumentando, assim, a demanda metabólica nos tecidos locais (Hou et al., 2002). Ademais, a sobrecarga de um músculo ou de um grupo específico de fibras musculares tal como ocorre quando se adquire hábitos posturais inadequados (microtrauma) ou quando se sofre um acidente (macrotrauma), pode acarretar em dano aos componentes intracelulares dessas fibras.

Além disso, os trigger points podem se desenvolver em resposta a outros estímulos nocivos ao músculo afetado, tais como: infecções, estados febris, dores profundas, distúrbios hormonais, distúrbios viscerais, distúrbios neuro-humorais (fadiga, fibromialgia), fatores ambientais, distúrbios esqueléticos intrínsecos (síndromes dolorosas miofasciais) (Aftimos, 1989). Pode-se citar ainda baixo condicionamento físico, avitaminoses e distúrbios do sono.

Os pacientes que apresentam trigger points freqüentemente relatam dor regional persistente, que muitas vezes resulta em diminuição da amplitude do movimento do músculo afetado (Alvarez e Rockwell, 2002). Ador é de difícil localização, sentida nos tecidos subcutâneos ou na articulação. Entre as queixas mais comuns que os pacientes odontológicos relatam ao se apresentarem para tratamento de dor estão a dor de cabeça tipo tensional, dor no seio maxilar e odontalgias. O adormecimento da área ou parestesia podem ocorrer ao invés da dor. Podem estar presentes, ainda, zumbido, dor na articulação temporomandibular, sintomas oculares e torcicolo (Alvarez e Rockwell, 2002). Menos comumente podem ocorrer náuseas e vertigem. Efeitos autonômicos associados aos trigger points incluem sudoreze anormal, lacrimejamento constante, coriza nasal persistente e salivação excessiva.

Como já foi dito, a DMF referida origina-se de trigger points, em um músculo. Entretanto a localização da dor não ocorre casualmente, mas sim em um padrão específico, característico de cada músculo (Padamsee et al., 1987). Os padrões de referência de dor foram identificados e mapeados e sugere-se a consulta a esses mapeamentos que são um guia valioso para identificação da fonte de dor. O achado clínico mais comum é que a origem e o local da dor compartilhem a mesma raiz nervosa, isto é, sejam ramos de um mesmo tronco nervoso (Travell e Simons, 1999).

Além disso, outra característica que deve estar presente na DMF é o fato de que quando os trigger points forem estimulados (palpação compressiva), eles deverão apresentar uma resposta de contração tran- 
sitória local das fibras musculares afetadas (local twitch response). A local twitch response pode acontecer também em resposta à penetração de uma agulha diretamente no trigger point (Alvarez e Rockwell, 2002). Algumas vezes a local twitch response é intensa a ponto de movimentar a parte do corpo que se relaciona com o músculo afetado. Esse fenômeno não deve ser confundido com um outro sinal característico de um triger point sob compressão: o chamado "sinal do pulo" (jump sign). O jump sign trata-se do movimento de esquiva do paciente em conseqüência da dor provocada pelo estímulo de um trigger point. Muitas vezes a compressão de um trigger point provoca alterações na expressão facial e até mesmo lágrimas no paciente (Travell e Simons, 1999).

O aumento da tensão muscular, o encurtamento e a diminuição da capacidade de alongamento normal do músculo podem estar presentes, principalmente em presença de trigger points latentes. A contração de um músculo que se apresenta tenso ou encurtado pode facilitar a ativação dos trigger points latentes. Podem ocorrer também fraqueza muscular e baixa resistência ao esforço contínuo do músculo afetado (Travell e Simons, 1999).

O clínico deverá estar apto a localizar o trigger point pela palpação. Quando um músculo é acometido pela presença de trigger points, um conjunto de fibras musculares encontra-se, atipicamente, com sua região central bastante contraída, apresentando os sarcômeros muito próximos entre si, formando uma região nodular (nó de contração). As extremidades dessas fibras encontram-se estiradas, afilando-se com o afastamento dos sarcômeros uns dos outros. Vários nós de contração próximos formam um trigger point central. A extensão total das fibras acometidas torna-se muito tensa e é isto que promove a formação da banda rígida (Travell e Simons, 1999).

O mecanismo pelo qual se formam os trigger points está por ser completamente esclarecido. Alguns autores sugerem que o dano ao músculo ocorre primariamente no nível das placas motoras, onde se cria uma crise de energia. De acordo com Fricton (Fricton, 1999), essa crise se dá pelo aumento progressivo no metabolismo oxidativo, principalmente nas fibras musculares do tipo I que estejam com suprimento de energia deficiente, aumento da produção de metabólitos e conseqüente nocicepção muscular na periferia. Isso resulta em dor local e referida pelo sistema nervoso central, passível de ser alterada por um mecanismo central, que pode tanto perpetuar, quanto suprimir a dor.

As fibras do tipo I são grossas fibras musculares de contração lenta, associadas à manutenção do tônus muscular. Essas fibras requerem um grande número de mitocôndrias para a realização da fosforilação oxidativa usada no metabolismo energético, uma vez que não utilizam as reservas energéticas na forma de glicogênio (tais como as fortes fibras de contração rápida do tipo II). Dependendo do tipo de demanda muscular, essas fibras podem se transformar de umas para as outras. Dessa forma, um aumento da demanda de atividade muscular postural pode aumentar o número de fibras tipo I e diminuir as fibras tipo II. Entretanto, se essas fibras são exigidas além dos limites normais (como quando da presença de hábitos parafuncionais), pode haver excesso de liberação de acetilcolina, a qual é um mediador químico liberado no terminal pós-sináptico das placas motoras, que promove a contração muscular pela liberação de íons cálcio.

A contração freqüente provoca um aumento na demanda energética, contração dos vasos sanguíneos da região e aumento da liberação de metabólitos. Além disso, esse processo pode causar dano aos componentes intracelulares das fibras musculares com hiperpolarização externa do músculo devido a altos níveis de $\mathrm{K}^{+}$provocados por atividade motora sustentada $\mathrm{e}$ dano à bomba de $\mathrm{K}^{+}$, dano aos filamentos de actina $\mathrm{e}$ miosina, rompimento do retículo sarcoplasmático, dano à bomba de cálcio e a diminuição do fluxo sangüíneo. Como conseqüência, há um acúmulo de metabólitos na região, entre as quais muitas substâncias neurotransmissoras, que podem sensitizar o sistema nervoso central, desencadeando mais estímulos de contração no local e/ou em áreas de referência, perpetuando o ciclo e provocando a formação dos trigger points. Dessa forma, os músculos acometidos pelos trigger points e outros relacionados a eles podem apresentar sua posição postural modificada devido à dor provocada pelo alongamento muscular. Essa ausência de repouso provoca o estresse muscular acarretando a formação de novos trigger points. Esse mecanismo de retroalimentação leva muitas vezes à cronificação 
da doença, tornando mais difícil o tratamento.

Dessa forma, a DMF é produzida, exacerbada ou perpetuada principalmente por fatores que danifiquem ou provoquem o estresse dos músculos esqueléticos, tais como determinados hábitos parafuncionais orais e posturais inadequados, durante o sono e vigília. Por isso tem-se afirmado que os trigger points podem se desenvolver ou serem ativados a partir de macro ou microtraumas aos músculos, estados febris, distúrbios esqueléticos, neuro-humorais ou vicerais, avitaminoses, baixa qualidade de sono e depressão. Fatores que levam a má postura como obesidade, sedentarismo, falta de exercícios e falta de planejamento (ergonomia) de mobílias e equipamentos de trabalho também podem levar ao aparecimento ou ativação de trigger points. Um dos traumas que os músculos freqüentemente sofrem são aqueles provocados por acidentes. Um movimento brusco e violento, tal como ocorre durante uma batida de automóvel, por exemplo, pode promover o estiramento muscular e é comum, nessa situação, que ocorra a co-contração protetora. Enquanto persistir essa desordem, o movimento muscular promoverá dor, e essa dor poderá provocar o aparecimento de trigger points.

Os hábitos parafuncionais e a má postura durante trabalho são fatores que podem causar microtraumas musculares. Durante essas atividades, determinada fibras musculares são constantemente contraídas, sobrecarregando-se. Essas fibras podem entrar em fadiga, criando as condições propícias ao aparecimento dos trigger points. Além disso, toda a atividade muscular realizada durante as atividades normais do ser humano exige um período de reparação para os tecidos musculares. As fases 3 e 4 do sono não-REM estão fortemente relacionadas com essa recuperação (Schaefer, 2003). Quando se suprime essa fase do sono, ou quando o paciente dorme mal, independentemente do motivo, ele estará mais propenso a desenvolver a DMF. Ademais, tem-se investigado a influência de fatores hormonais, infecções e fontes de dores profundas no seu desenvolvimento e ativação (Aftimos, 1989; Dao et al., 1998; Davidoff, 1998; Fricton, 1999; Carneiro, 2002).

De acordo com Fricton (1999), atividades nociceptivas centrais ou periféricas prolongadas podem resultar em alterações neuroplásticas, tais como a res- ponsividade prolongada a impulsos aferentes, aumento da amplitude do campo receptivo e atividades espontâneas de dor em neurônios superficiais ou profundos. Essa condição dolorosa do músculo pode reforçar hábitos posturais inadequados que previnam a atividade muscular funcional aumentando sua tensão e conseqüentemente provocando mais dor. Dao et al.(1998) investigaram a flutuação da dor ocasionada pela presença de DMF, em função dos ciclos hormonais de mulheres com ciclos menstruais normais e mulheres que faziam uso de contraceptivos orais. Os autores verificaram que as pacientes com ciclos menstruais normais apresentaram períodos sem dor, enquanto que aquelas que faziam uso de contraceptivos orais, não. Raphael et al. (1999) em um estudo familial, descartaram a hipótese da ocorrência significativa de DMF em parentes de primeiro grau de pacientes acometidos por DMF. Entretanto, os autores utilizaram um critério de inclusão no diagnóstico da DMF que não condiz com aquele utilizado nesse texto, que se baseia na percepção de trigger points à palpação, dor referida, local twitch response e jump sign.

Os trigger points podem ser classificados, segundo suas características clínicas, em ativos ou latentes. Um trigger point ativo causa dor, mesmo com o músculo em repouso. Ele é dolorido à palpação e acarreta também dor referida com padrão similar à dor descrita pelo paciente. Essa dor é freqüentemente descrita como esparramada ou irradiada. Um trigger point latente não causa dor espontânea, apesar de poder ser a causa de restrição motora ou fraqueza muscular. Nesse caso, o paciente pode tomar conhecimento da dor provocada por um trigger point somente por meio da aplicação direta de força compressiva sobre ele. Os trigger points podem ser classificados também, segundo sua localização dentro do músculo afetado, em centrais ou de fixação (attachment) e de acordo com sua origem, em primários, secundários ou satélites (Travell e Simons, 1999).

\section{Tratamento}

Para o tratamento da DMF, Davidof (1998) sugere três tipos de abordagem: injeção de solução anestésica nos trigger points, fisioterapia (aplicação 
de bolsas quentes, ultrasom e massagem terapêutica para possibilitar o alongamento muscular) e controle dos fatores perpetuantes, principalmente no que se refere à má postura muscular. De acordo com Nicolakis et al. (2002), o tratamento fisioterápico pode contribuir para o tratamento da "síndrome" da DMF, termo utilizado pelo autor como genérico, que inclui diversas desordens temporomandibulares.

Borg-Stein e Simons (2002) incluíram várias modalidades de tratamento para a DMF. Para controle dos fatores e desordens associados o autor sugere: farmacoterapia (agentes antinflamatórios não esteroidais, Tramadol, anti-depressivos, agonistas alfa-adrenérgicos, anticonvulsivantes e toxina botulínica), modificações de postura, redução do estresse, acupuntura, massagem, TENS, ultrasom, fisioterapia para alongamento muscular e injeção local nos trigger points. Alvarez e Rockwell (2002) enfatizam a importância do controle dos fatores perpetuantes, o uso de terapia farmacológica para as dores crônicas e a eliminação local dos trigger points pela fisioterapia dos músculos afetados. O uso de toxina botulínica, que inibe a contração muscular pelo bloqueio da liberação de acetilcolina nos nervos periféricos, foi efetivo para o tratamento da DMF e foi indicado para o tratamento da mesma. A redução da DMF e o aumento da amplitude de movimento ativo dos músculos esqueléticos foram conseguidos através da utilização de tratamento fisioterápico (Hou et al., 2002).

Raphael et al. (2003) confeccionaram placas miorelaxantes para pacientes que apresentavam bruxismo e DMF nos músculos da face e compararam com placas placebo. Os autores observaram que apesar dos índices de dor terem sido diminuídos nos pacientes tratados com a placa miorelaxante ativa, o bruxismo não foi diminuído nesses pacientes.

Entretanto, em pacientes que apresentem trigger points nas regiões dos músculos mastigatórios, como temporal e masséter, o controle dos danos da atividade parafuncional através do uso de uma placa oclusal, ainda que o bruxismo não seja diminuído, parece clinicamente ser de extrema importância para o prognóstico do caso.

Uma das formas mais importantes de abordagem terapêutica que deve ser instituída para a DMF é a educação do paciente e mudanças comportamentais. O paciente deve estar consciente do seu problema e dos fatores que o causam, aumentam ou perpetuam a DMF. As mudanças comportamentais visam à eliminação ou o controle desses fatores, por meio de correção de hábitos posturais inadequados; melhoria da alimentação, evitando-se episódios de hipoglicemia e a ingestão de cafeína, aumentando-se a ingestão de líquidos e evitando-se avitaminoses; eliminação de hábitos parafuncionais; prática de exercícios físicos aeróbicos para melhoria do condicionamento físico; instituição de terapia psicológica e relaxamento, caso se verifique que o estresse emocional pode causar ou colaborar para a tensão muscular e instrução de respiração diafragmática.

Além disso, pode-se lançar mão do uso de analgésicos: Advil (Ibuprofeno); relaxantes musculares: Miosan(Ciclopenzapina) ou Sirdalud (Tizanidina); antidepressivos tricíclicos: Pamelor (Nortriptilina).

Outra forma de tratamento indicada é a fisioterapia caseira, através de exercícios de alongamento passivo, que deve ser realizada em ausência de dor. Para tanto, deve ser previamente utilizada a aplicação de gelo na região durante 5 minutos. Após alongados, os músculos podem receber compressas quentes de 15 a 20 minutos para promover o relaxamento.

Da mesma forma, a fisioterapia assistida por profissional da área consiste de exercícios de tensão isométrica suave contra resistência; movimentos de abertura e fechamentos guiados; distração articular guiada; alongamento muscular precedido ou não de vaporização (gás fluormetano ou éter de etila) ou aplicação de gelo; TENS; massagem; mobilização do complexo côndilo-disco e correção da postura e técnicas de relaxamento.

Uma das modalidades de tratamento mais efetivas para a eliminação de trigger points, são as injeções (Padamsee et al., 1987; Ivawa, 2001; Alvarez e Rockwell, 2002; Irnich et al., 2002). Essa forma de tratamento somente pode ser utilizada quando o trigger point estiver ativo e/ou reproduzindo a queixa do paciente, na ocasião da sessão de injeção.

O princípio de ação das injeções não foi totalmente esclarecido e existem algumas teorias que tentam explicá-lo: rompimento mecânico das fibras musculares e terminais nervosos; rompimento mecânico das fibras musculares causando aumento do potássio extracelular, o que leva à despolarização dos nervos; interrupção 
do mecanismo de feedback positivo que perpetua a dor; diluição das substâncias nociceptivas pelo líquido infiltrado; efeito vasodilatador do anestésico que aumenta a remoção dos metabólitos. As injeções podem ser realizadas de diversas maneiras, de acordo com o tipo de solução injetada: somente agulha (agulhamento a seco), injeção de solução salina, injeção de soluções anestésicas: lidocaína a $0,25 \%$, mepivacaína a $0,2 \%$ (Iwama et al., 2001), xilocaína sem vasoconstrictor (Padamsee et al., 1987), procaína a 0,5\% sem vasoconstrictor (Travell e Simons, 1999) e injeção de toxina botulínica. Qualquer que seja o líquido injetado, as características da agulha são um importante fator a observar: essas não devem ser tão finas a ponto de dobrar ao contatar o trigger point e nem tão grossas a ponto de produzir uma lesão muito grande com conseqüente dolorimento local. As agulhas mais grossas permitem maior sensibilidade sobre o tipo de tecido que se está perfurando.

Entre os tratamentos alternativos para a DMF, poderíamos citar a acupuntura, amplamente discutida por Carneiro (Carneiro, 2002).

\section{Discussão}

\section{DMF versus FM}

O diagnóstico da FM muito freqüentemente é negligenciado pelos profissionais de saúde, em função de outras doenças que apresentam sintomas similares. Assim, antes de considerarmos qualquer opção terapêutica um diagnóstico definitivo deve ser estabelecido.

Dentre as doenças relacionadas com a área odontológica, a DMF pode ser confundida e/ou coexistir com a FM, em muitos pacientes (Wolfe, 1990). Plesh et al. (1996) analisaram e compararam pacientes com DMF e FM observando que uma pequena porcentagem de pacientes com DMF $(18,4 \%)$ apresentou FM, mas muitos dos pacientes com FM ( $75 \%$ ) apresentaram DMF. Isto ocorre devido à presença de algumas características clínicas em comum em ambas as patologias, tais como a presença de dor, sono não reparador, depressão, má postura, sedentarismo e ansiedade, entre outros (Yunus et al. 1988; Travell e Simons, 1999; Alencar Jr, 2002; Ferucci e Collier, 2002;
Mehendale e Goldman, 2002). Além disso, é importante ressaltar que os pacientes com FM e DMF apresentam alteração no processamento da percepção da dor, fenômeno relacionado à neuroplasticidade e à sensitização do SNC (Travell e Simons, 1999; Staud e Domingo, 2001). No caso específico da DMF, o acometimento do SNC é secundário a uma alteração muscular e regional. Entretanto, ao contrário do que se acreditava, a FM não é de origem muscular, mas sim de origem no SNC. Isto explica sua distribuição generalizada e simétrica no corpo humano.

O diagnóstico da FMe da DMF é essencialmente clínico e eliminatório. Portanto, a realização de exame clínico detalhado (anamnese e exame físico) é de fundamental importância para o correto diagnóstico. Entre os critérios diagnóstico da FM, a presença de pontos dolorosos é condição obrigatória para o diagnóstico de síndrome fibromiálgica (Ferucci e Collier, 2002; McCarberg et al., 2003). Porém, é importante ressaltar a diferença entre os pontos dolorosos e os trigger points.

Os trigger points, presentes na DMF, são caracterizados pela presença de bandas enrijecidas, dor referida, resposta de contração à palpação e o "sinal do pulo" (esquiva) (Travell e Simons, 1999). Essas características não devem ser observadas durante a palpação dos pontos dolorosos que, clinicamente, apresentam-se como áreas doloridas locais de distribuição generalizada e simétrica, porém sem padrão de dor referida (Ferucci e Collier, 2002).

Apesar das diferentes formas de tratamento, não há uma terapia única indicada para todos os sintomas de todos os pacientes com FM e/ou DMF. Além disso, algumas formas de tratamento como, por exemplo, o agulhamento, indicadas para a DMF são contraindicadas para a FM e vice-versa. Para os pacientes que possuem FM e DMF, o tratamento deve ser multidisciplinar, com o apoio de diversos profissionais da saúde (fisioterapeuta, terapeuta ocupacional, enfermeiro, psicólogo, médico e cirurgião-dentista) e com a associação das diversas modalidades terapêuticas (educação, recondicionamento físico, condicionamento do trabalho, aconselhamento e terapia farmacológica) (Cheshire et al., 1994; Davidoff, 1998; Alvarez e Rockwell, 2002; Bor-Stein e Simons, 2002; Sprott, 2003). 


\section{Conclusão}

A FM é uma doença crônica e complexa, caracterizada pelo desconforto músculo-esqueletal generalizado e alterações nos sistemas nervoso central, endócrino e psicológico. Dentro desse contexto, o cirurgião-dentista exerce uma importante função no diagnóstico e tratamento da FM, visto que essa síndrome pode coexistir com outras doenças que acometem a região de cabeça e pescoço tal como a DMF. Portanto, esses profissionais devem estar preparados para fazer o diagnóstico de ambas as doenças de forma adequada, evitando intervenções odontológicas desnecessárias. Além disso, devido ao acometimento do SNC, a FM pode perpetuar a DMF bem como outras desordens temporomandibulares coexistentes. Assim, a instituição de diversas modalidades terapêuticas associadas à participação de uma equipe multidisciplinar devem ser procedimentos de rotina para que seja obtido o sucesso no tratamento da FM e das desordens associadas, em especial a DMF.

\section{REFERÊNCIAS}

1. AFTIMOS, S. Myofascial pain in children. The New Zealand Medical Journal, Wellington, v. 23, n. 102(874), p. 440-441, 1989.

2. ALENCAR Jr., F. G. P. Effectiveness of relaxin in the management of fibromyalgia patients. Minnesota, 2002. Dissertação (Mestrado em) - University of Minnesota.

3. ALVAREZ, D. J. e ROCKWELL, P. G. Trigger points: diagnosis and management. American Family Physician, Kansas City, v. 15, n. 65(4), p. 653-660, 2002.

4. BORG-STEIN, J. e SIMONS, D. G. Focused review: myofascial pain. Archives of Physical Medicine and Rehabilitation, Philadelphia, v. 83, n. 3 (Suppl 1), p. S40-7 - S48-9, 2002.

5. BUSKILA, D. Fibromyalgia: a biopsychosocial syndrome. The Israel Medical Association Journal, Ramat Gan, v. 5, n. 12,p. 887-888, 2003

6. CARNEIRO, N. M. Acupuntura no tratamento da dor myofascial. São Paulo: Associação Médica Brasileira. Conselho Federal De Medicina. Projeto diretrizes, 2001. 13 p.

7. CHEShIRE, W. P., ABASHIAN, S. W., MANN, J. D. Botulinum toxin in the treatment of myofascial pain syndrome. Pain, Amsterdam, v. 59, n. 1, p. 65-69, 1994.

8. DAO, T. T., KNIGHT, K., TON-THAT, V. Modulation of myofascial pain by the reproductive hormones: a preliminary report. The Journal of Prosthetic Dentistry, St Louis, v. 79, n. 6, p. 663-670, 1998.

9. DAVIDOFF, R. A. Trigger points and myofascial pain: toward understanding how they affect headaches. Cephalalgia: an International Journal of Headache, Oslo, v. 18, n. 7, p. 436448, 1998 .

10. Dicionário de Especialidades Farmacêuticas (DEF) 2001/ 02. Jornal Brasileiro de Medicina, $31^{\text {th }}$ ed. Rio de Janeiro: Editora de Publicações Científicas Ltda, 2001.

11. FERUCCI, E. e COLLIER, D. H. Fibromyalgia: Diagnosis and Treatment. Primary Care Case Reviews. 2002; 5(3):101-8.

12. FORSETH, K. K. O. e GRAN, J. T. Management of fibromyalgia: what are the best treatment choices? Drugs, New York, v. 62, n. 4, p. 577-592, 2002.

13. FRICTON, J. R. Masticatory myofascial pain: an explanatory model integrating clinical, epidemiological and basic science research. Bulletin du Groupement International pour la Recherche Scientifique en Stomatologie \& Odontologie, Bruxelas, v. 41, n. 1, p. 14-25, 1999.

14. GEENEN, R. e JACOBS, J. W. G. Fibromyalgia: diagnosis, pathogenesis, and treatment. Current Opinion in Anaesthesiology, Philadelphia, v. 14, n. 5, p. 533-539, 2001.

15. HAN, S. C. e HARRISON, P. Myofascial pain syndrome and trigger-point management. Regional anesthesia, Greenwich, v. 22, n. 1, p. 89-101, 1997.

16. HOU, C. R., TSAI, L. C., CHENG, K. F., CHUNG, K. C., HONG, C. Z. Immediate effects of various physical therapeutic modalities on cervical myofascial pain and trigger-point sensitivity. Archives of Physical Medicine and Rehabilitation, Chicago, v. 83, n. 10, p. 1406-1414, 2002.

17. IRNICH, D., BEHRENS, N., GLEDITSCH, J. M., STOR, W., SCHREIBER, M. A., SCHOPS, P. et al. Immediate effects of dry needling and acupuncture at distant points in chronic neck pain: results of a randomized, double-blind, sham-controlled crossover trial. Pain, Amsterdam, v. 99, n. 1-2, p. 83-89, 2002.

18. IWAMA, H., OHMORI, S., KANEKO, T., WATANABE, K. Water-diluted local anesthetic for trigger-point injection in chronic myofascial pain syndrome: evaluation of types of local anesthetic and concentrations in water. Regional Anesthesia and Pain Medicine, Secaucus, v. 26, n. 4, p. 333-336, 2001.

19. JONES, K. D., CLARK, S. R., BENNETT, R. M. Prescribing exercise for people with fibromyalgia. AACN Clinical Issues, Philadelphia, v. 13, n. 2, p. 277-293, 2002.

20. LEVENTHAL, L. J. Management of fibromyalgia. Annals of Internal Medicine, Philadelphia, v. 131, n. 11, p. 850-858, 1999.

21. MCCARBERG, B., BARKIN, R. L., WRIGHT, J.A., CRONAN, T. A., GROESSL, E., SCHMIDT, S. M. Tender points as predictors of distress and the pharmacologic management of 
fibromyalgia syndrome. American Journal of Therapeutics, New York, v. 10, n. 3, p. 176-192, 2003.

22. MEHENDALE, A. W., GOLDMAN, M. P. Fybromyalgia syndrome, idiophatic widespread persistent pain or syndrome of myalgic encephalomyelopathy (SME): what is its nature? Pain practice : the official journal of World Institute of Pain, Malden, v. 2, n. 1, p. 35-46, 2002.

23. MOLDOFSKY, H. Management of sleep disorders in fibromyalgia. Rheumatic diseases clinics of North America, Philadelphia, v. 28, n. 2, p. 353-365, 2002.

24. NEECK, G. Pathogenic mechanisms of fibromyalgia. Ageing research reviews, Oxford; v. 1, n. 2, p. 243-255, 2002.

25. NICOLAKIS, P., ERDOGMUS, B., KOPF,A., NICOLAKIS, M., PIEHSLINGER, E., FIALKA-MOSER, V. Effectiveness of exercise therapy in patients with myofascial pain dysfunction syndrome. Journal of Oral Rehabilitation, Oxford, v. 29, n. 4, p. 362-368, 2002.

26. OFFENBAECHER, M., BONDY, B., DE JONGE, S., GLATZEDER, K., KRUGER, M., SCHOEPS, P. et al. Possible association of fibromyalgia with a polymorphism in the serotonin transporter gene regulatory region. Arthritis and Rheumatism, Atlanta, v. 42, n. 11, p. 2482-2488, 1999.

27. PADAMSEE, M., MEHTA, N., WHITE, G. E. Trigger point injection: a neglected modality in the treatment of TMJ dysfunction. The Journal of Pedodontics, Birmingham, v. 12, n. 1, p. 72-92, 1987.

28. PLESH, O., WOLFE, F., LANE, N. The relationship between fibromyalgia and temporomandibular disorders: prevalence and symptom severity. The Journal of Rheumatology, Toronto, v. 23, n. 11, p. 1948-1952, 1996.

29. RAPHAEL, K. G., MARBACH, J. J., GALLAGHER, R. M., DOHRENWEND, B. P. Myofascial TMD does not run in families. Pain, Amsterdam, v. 80, n. 1-2, p.15-22, 1999.

30. RAPHAEL, K. G., MARBACH, J. J., KLAUSNER, J. J., TEAFORD, M. F., FISCHOFF, D. K. Is bruxism severity a predictor of oral splint efficacy in patients with myofascial face pain? Journal of Oral Rehabilitation, Oxford, v. 30, n. 1, p. 17-29, 2003.

31. RUSSEL, M. Current status of genetic discoveries in cluster headache. Italian Journal of Neurological Sciences, Milão, v. 20, n. 2 Suppl, p. S7-9, 1999.
32. SCHAEFER, K. M. Sleep disturbances linked to fibromyalgia. Holistic Nursing Practice, Frederick, v. 17, n. 3, p. 120-127, 2003.

33. SIMONS, D. G. Fibrositis/fibromyalgia: a form of myofascial trigger points? The American Journal of Medicine, New York, v. 29, n. 81-3A, p. 93-98, 1986.

34. SPROTT, H. What can rehabilitation interventions achieve in patients with primary fibromyalgia? Current Opinion in Rheumatology, Philadelphia, v. 15, n. 2, p. 145-150, 2003.

35. STAUD, R. e DOMINGO, M. Evidence for abnormal pain processing in fibromyalgia syndrome. Pain medicine: the official journal of the American Academy of Pain Medicine, Malden, v. 2, n. 3, p. 208-215, 2001.

36. THANT, Z. S. e TAN, E. K. Emerging therapeutic applications of botulinum toxin. Medical science monitor: international medical journal of experimental and clinical research, Warsaw, v. 9, n. 2, p. RA40-8, 2003.

37. TRAVELL, J. S. e SIMONS, L. S. Travell \& Simons' myofascial pain and dysfunction. The trigger point manual. $2^{\text {th }}$ ed. Baltimore: Williams \& Wilkins; 1999, 1038 p.

38. TUZUN, E. H., ALBAYRAK, G., EKER, L., SOZAY, S., DASKAPAN, A. A comparison study of quality of life in women with fibromyalgia and myofascial pain syndrome. Disability and Rehabilitation, London, v. 18, n. 26, p. 198-202, 2004.

39. WOLFE, F., SMYTHE, H.A., YUNUS, M. B., BENNETT, R. M., BOMBARDIER, C., GOLDENBERG, D. L., TUGWELL, P., CAMPBELL, S. M., ABELES, M., CLARK, P. et al. The American College of Rheumatology 1990 Criteria for the Classification of Fibromyalgia. Report of the Multicenter Criteria Committee. Arthritis and Rheumatism, Atlanta, v. 33, n. 2, p. 160-172, 1990.

40. YUNUS, M. B., KALYAN-RAMAN, U. P., KALYANRAMAN, K. Primary fibromyalgia syndrome and myofascial pain syndrome: clinical features and muscle pathology. Archives of physical medicine and rehabilitation, Chicago, v. 69, n. 6, p. 451-454, 1988.

41. YUNUS, M. B. Psychological aspects of fibromyalgia syndrome: a component of the dysfunctional spectrum syndrome. Baillieİre's clinical rheumatology, London, v. 8, n. 4,p. 811-837, 1994. 